\title{
Multiple Neuroendocrine Tumors in Stomach and Duodenum in a Multiple Endocrine Neoplasia Type 1 Patient
}

\author{
Bohyun Kim · Han-Kwang Yang ${ }^{1}$ \\ Woo Ho Kim \\ Departments of Pathology and ${ }^{1}$ Surgery, Seoul \\ National University College of Medicine, Seoul, \\ Korea \\ Received: June 19, 2017 \\ Revised: September 14, 2017 \\ Accepted: September 16, 2017 \\ Corresponding Author \\ Woo Ho Kim, MD, PhD \\ Department of Pathology, Seoul National University \\ College of Medicine, 103 Daehak-ro, Jongno-gu, \\ Seoul 03080, Korea \\ Tel: +82-2-740-8269 \\ Fax: +82-2-765-5600 \\ E-mail:woohokim@snu.ac.k
}

\begin{abstract}
A 67-year-old woman with a history of subtotal parathyroidectomy, distal pancreatectomy, and total splenectomy 23 years prior underwent surgical gastric resection for neuroendocrine tumors of the stomach and duodenum. Meticulous examination of the entire stomach and duodenum revealed multiple scattered, minute neuroendocrine tumors. To the best of our knowledge, this is the first case report of a patient diagnosed with gastroduodenal neuroendocrine tumors associated with multiple endocrine neoplasia type 1 (MEN 1) in whom complete histologic mapping of the whole gastrectomy specimen was performed. The presence of MEN 1-associated neuroendocrine tumors in the stomach is very rare, but should be considered in patients diagnosed with MEN 1 who present with a new tumor in the stomach.
\end{abstract}

Key Words: Multiple endocrine neoplasia type 1; Stomach neoplasms; Neuroendocrine tumors; Histologic mapping
Multiple endocrine neoplasia (MEN) is a rare, autosomaldominant disease caused by a genetic mutation and shows a familial tendency. ${ }^{1,2}$ Various kinds of endocrine glands may be involved in this condition. According to the affected genes and the distribution of neuroendocrine tumor-involved organs, MEN is classified into types $1,2,3$, and $4 .{ }^{3}$ MEN 1 has a prevalence of $0.25 \%$ in postmortem studies, and is found in patients of all ages. ${ }^{4}$ MEN 1 can be diagnosed based on three different criteria. First, a clinical diagnosis can be made if two or more MEN 1-associated tumors are found. Second, a familial diagnosis can be made if a MEN 1 patient who has been clinically diagnosed has a first-degree family member with a history of MEN 1. Finally, a genetic diagnosis can be made if a MEN 1 germ-line mutation is confirmed, even in the absence of clinical and biochemical manifestations. ${ }^{5}$

\section{CASE REPORT}

A 67-year-old woman visited Seoul National University Hospital after an abnormal finding was detected on esophagogastroduodenoscopy (EGD) performed as part of a routine medical examination at another hospital. She previously underwent right thyroidectomy with subtotal parathyroidectomy, distal pancreatectomy, and total splenectomy 23 years prior. Pathological evaluation at that time resulted in the diagnosis of parathyroid gland hyperplasia, thyroid adenomatous goiter, and pancreas islet cell adenomas. The clinical diagnosis was MEN 1. The patient's younger sister was also diagnosed with MEN 1.

Laboratory studies from 23 years prior showed preoperative and postoperative serum gastrin levels of 222 and $143 \mathrm{ng} / \mathrm{dL}$ (normal range, $<90 \mathrm{ng} / \mathrm{dL}$ ), respectively, and calcium levels of 10.7 and $7.9 \mathrm{mg} / \mathrm{dL}$ (normal range, 8.4 to $10.2 \mathrm{mg} / \mathrm{dL}$ ), respectively. Thus, the patient was clinically diagnosed with MEN 1. One year prior, two adenocarcinoma lesions were found on the posterior wall of the stomach antrum, and they were successfully removed via endoscopic submucosal dissection.

The patient underwent EGD, which revealed a subepithelial mass with an approximate size of $0.5 \mathrm{~cm}$ in the stomach cardia and $1 \mathrm{~cm}$ sized multiple subepithelial masses in the second portion of the duodenum (Fig. 1A). Biopsy was performed and immunohistochemical evaluation revealed tumor cells positive for chromogranin A, synaptophysin, and cytokeratin. The pathological diagnosis was neuroendocrine tumor for all lesions (Fig. 1B-D). 

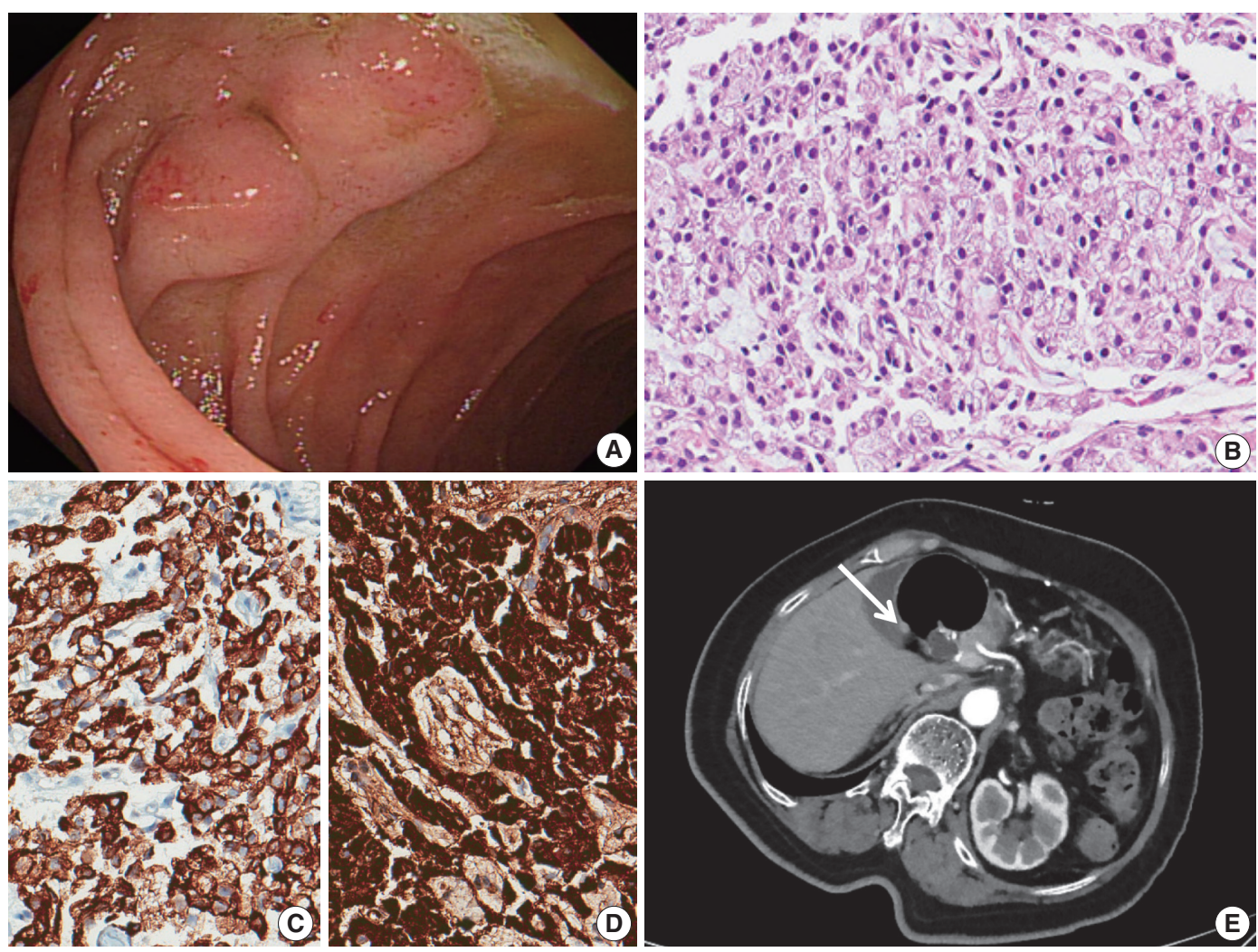

Fig. 1. (A) Two subepithelial masses were found in the duodenum on esophagogastroduodenoscopy. (B) A neuroendocrine tumor with a diffuse pattern. (C) Immunohistochemistry of cytokeratin. (D) Immunohistochemistry of chromogranin A. (E) Abdominal computed tomography reveals a 6-mm-sized nodule (arrow) in the duodenal bulb.

On three-dimensional computed tomography of the stomach, a definite subepithelial tumor could not be observed in the stomach cardia, but a 6-mm-sized subepithelial tumor was found in the duodenal bulb (Fig. 1E). Three additional tiny, hyperenhanced lesions were found in the uncinate process of the pancreas. Considering her medical history, the possibility of the lesions being neuroendocrine tumors could not be excluded. No lesions were found in the pituitary gland or adrenal gland.

Total gastrectomy with extended resection of the proximal duodenum was performed. On macroscopic examination, three polypoid lesions were identified in the duodenum. They consisted of multiple polypoid submucosal nodules covered by centrally umbilicated hyperemic mucosa, each with a size of less than 1 $\mathrm{cm}$. Polypoid lesions were macroscopically not visible in the stomach cardia, where the neuroendocrine tumor was diagnosed. To evaluate the lesion in the stomach cardia, which was confirmed on EGD, and identify additional neuroendocrine lesions in the stomach, the entire resected specimen was subjected to a histologic mapping procedure. Two hundred seventeen sections were mounted in 109 each formalin-fixed, paraffin-embedded blocks. Meticulous microscopic examination of the entire specimen revealed 14 neuroendocrine lesions in the duodenum and five neuroendocrine lesions in the anterior and posterior walls of the stomach cardia. Among the 14 lesions in the duodenum, 10 had the greatest dimension $>0.5 \mathrm{~mm}$ and the remaining four lesions represented neuroendocrine dysplasia with the greatest dimension $>150 \mu \mathrm{m}$ but $<500 \mu \mathrm{m}$ (Fig. 2A). ${ }^{6}$ All five lesions in the stomach had the greatest dimensions $>0.5 \mathrm{~mm}$. Among the 19 lesions, only three were identifiable on macroscopic examination, and these were located in the duodenum as multiple nodules.

Microscopically, the neuroendocrine tumors in this case showed histopathologic features that were identical to those of sporadic neuroendocrine tumors. Duodenal lesions were all located in the mucosa or submucosa, with the greatest lesion being $0.8 \times 0.8 \times 0.3 \mathrm{~cm}$ in size. All lesions in the stomach were located in the lamina propria or submucosa, with the size of the greatest lesion being $0.3 \times 0.2 \times 0.2 \mathrm{~cm}$ (Fig. 2B). Immunohistochemical analysis revealed that the neuroendocrine markers synaptophysin and chromogranin A were positive. Lymphatic invasion, vascular invasion, and perineural invasion were not observed. Thirty regional lymph nodes were identified, and metastasis was confirmed in one lymph node in station 3 (lymph 

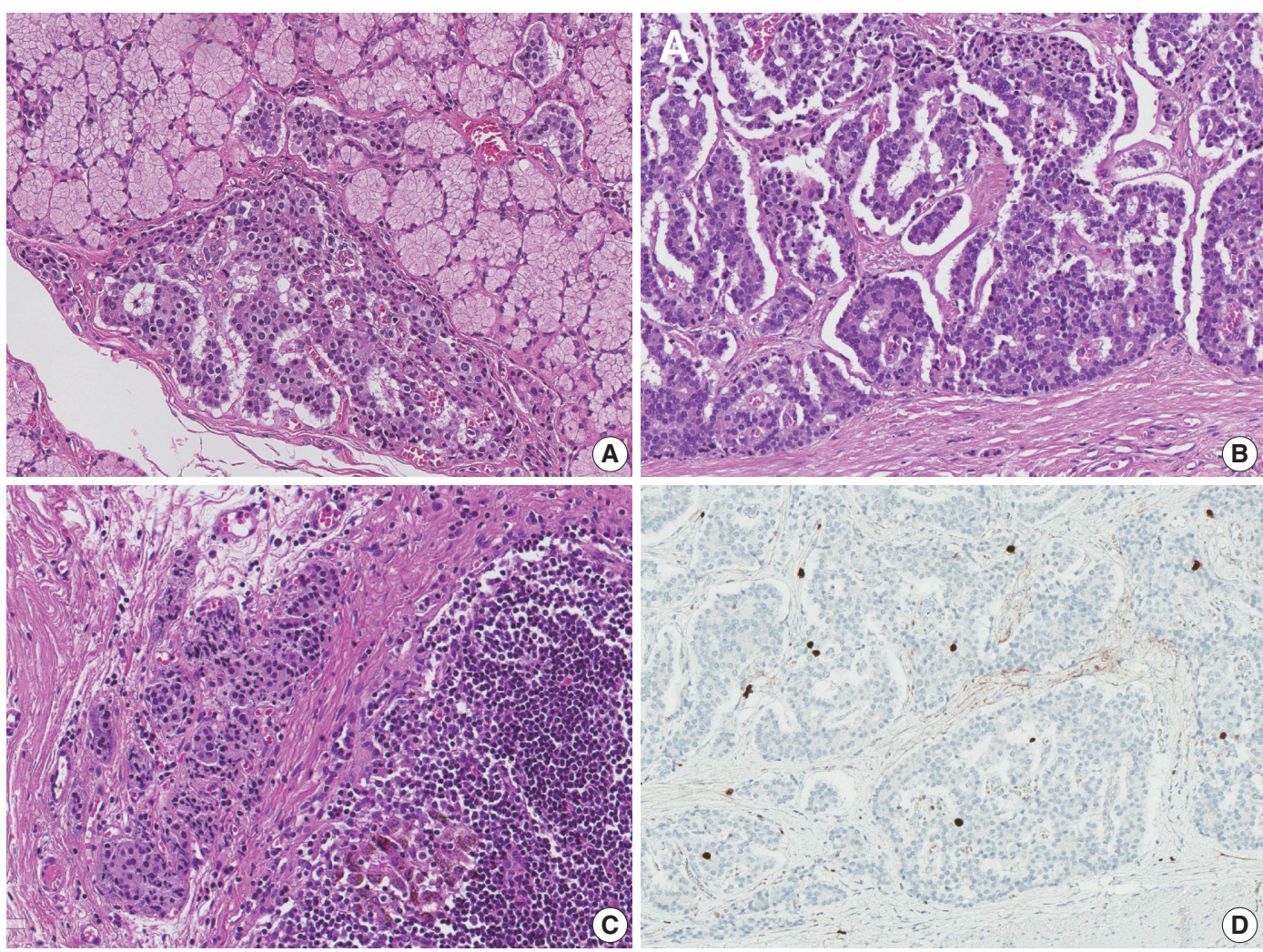

Fig. 2. Histologic features of neuroendocrine tumors. (A) Neuroendocrine dysplasia in the submucosal layer of the duodenum. (B) Neuroendocrine tumor in a ribbon-like growth pattern. (C) Metastatic lymph node in station 3. (D) Immunohistochemistry of Ki-67.

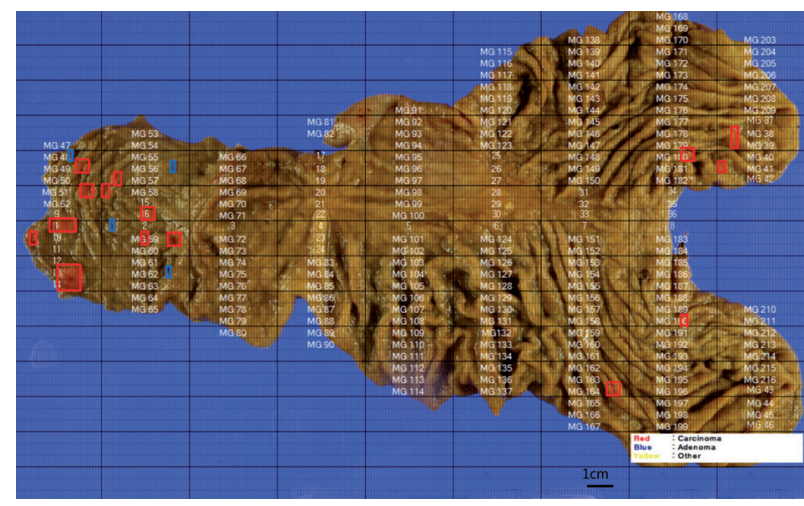

Fig. 3. Histologic mapping of the entire specimen revealed 19 neuroendocrine lesions in the stomach and duodenum (red, neuroendocrine tumor; blue, neuroendocrine dysplasia).

nodes along the lesser curvature). The size of the metastatic lymph node was $0.4 \mathrm{~mm}$ (Fig. 2C). On the histologic mapping procedure, 16 additional lesions were found (Fig. 3).

According to the 8th edition of the TNM staging system of the American Joint Committee on Cancer, neuroendocrine tumors of the stomach were stage $\mathrm{T} 1$, as they invaded the lamina propria or submucosa and were $\leq 1 \mathrm{~cm}$ in size. Neuroendocrine tumors in the duodenum were also stage $\mathrm{T} 1$, as they invaded only the mucosa or submucosa and were $\leq 1 \mathrm{~cm}$ in size. Both could be staged as $\mathrm{N} 1$ because of regional lymph node metastasis and as grade 1 , as they showed a Ki- 67 index of $<1 \%$ and mitotic activity of $<1 / 10$ high-power field (Fig. 2D).

The patient underwent follow-up abdominal computed tomography, and a small enhanced nodular lesion in the second portion of the duodenum and three enhanced lesions in the uncinated process of the pancreas were found. The possibility of additional neuroendocrine tumors could not be excluded. No recurrence was observed at the surgery site.

The study was approved by the Institutional Review Board of Seoul National University Hospital (IRB No. H-1706-098860 ) and performed in accordance with the principles of the Declaration of Helsinki. The informed consent was waived.

\section{DISCUSSION}

MEN 1 is a rare autosomal-dominant disease caused by a mutation in the MEN1 gene (chromosome 11) encoding the tumor suppressor protein menin. ${ }^{7,8}$ MEN 1 is suspected when two 
or more of the most common neuroendocrine tumors (parathyroid tumor, pancreatic islet cell tumor, and pituitary gland tumor) are found. Approximately $80 \%-90 \%$ of patients diagnosed with MEN 1 show a MEN1 gene mutation. ${ }^{9}$ However a genetic evaluation to confirm MEN1 gene mutation was not performed in this patient.

The incidence of gastrointestinal neuroendocrine tumors is increasing, and they account for $1 \%$ of all stomach tumors. ${ }^{10}$ Stomach neuroendocrine tumors can be subclassified into types I, II, and III. Type I neuroendocrine tumors accounts for $70 \%-$ $80 \%$ of stomach neuroendocrine tumors and primarily occur in patients with chronic atrophic gastritis, such as autoimmune gastritis and Helicobacter pylori-associated atrophic gastritis. Type III neuroendocrine tumors account for $10 \%-15 \%$ of stomach neuroendocrine tumors. These are sporadic tumors and are not associated with enterochromaffin cell hyperplasia. Finally, type II neuroendocrine tumors account for $5 \%-6 \%$ of stomach neuroendocrine tumors and are associated with MEN 1 and Zollinger-Ellision syndrome. In previous studies, 5\%-10\% of MEN 1 patients exhibited neuroendocrine tumors in the stomach, thymus, and lung. Type II neuroendocrine tumors are associated with hypergastrinemia, and they originate from enterochromaffin cells. $^{11,12}$

This report described a patient with a clinical diagnosis of MEN 1 and neuroendocrine tumor involvement of the stomach and duodenum, in whom whole-stomach histologic mapping was performed on a gastrectomy specimen. After mapping, multiple small lesions were detected that had not been observed on prior radiologic and macroscopic examinations.

In conclusion, we report a rare case of multiple gastric and duodenal neuroendocrine tumors associated with MEN 1. Histologic mapping was conducted using the entire resected specimen. This case shows that some patients with stomach neuroendocrine tumors associated with MEN 1 can develop multiple lesions in the stomach and duodenum, which can be overlooked on radiologic or endoscopic evaluation. Further studies are needed to assess the pathological characteristics of inherited stomach neuroendocrine tumors.

\section{Conflicts of Interest}

No potential conflict of interest relevant to this article was reported.

\section{REFERENCES}

1. Kouvaraki MA, Lee JE, Shapiro SE, et al. Genotype-phenotype analysis in multiple endocrine neoplasia type 1. Arch Surg 2002; 137: $641-7$.

2. de Laat JM, van der Luijt RB, Pieterman CR, et al. MEN1 redefined, a clinical comparison of mutation-positive and mutation-negative patients. BMC Med 2016; 14: 182.

3. Thakker RV. Multiple endocrine neoplasia type 1 (MEN1) and type 4 (MEN4). Mol Cell Endocrinol 2014; 386: 2-15.

4. Feliberti E, Perry RR, Vinik A. Multiple endocrine neoplasia type I and MEN II. In: De Groot LJ, Chrousos G, Dungan K, et al., eds. Endotext. South Dartmouth: MDText.com Inc., 2000.

5. Turner JJ, Christie PT, Pearce SH, Turnpenny PD, Thakker RV. Diagnostic challenges due to phenocopies: lessons from multiple endocrine neoplasia type1 (MEN1). Hum Mutat 2010; 31: E1089-101.

6. Grin A, Streutker CJ. Neuroendocrine tumors of the luminal gastrointestinal tract. Arch Pathol Lab Med 2015; 139: 750-6.

7. Wang GG, Yao JC, Worah S, et al. Comparison of genetic alterations in neuroendocrine tumors: frequent loss of chromosome 18 in ileal carcinoid tumors. Mod Pathol 2005; 18: 1079-87.

8. Christopoulos C, Papavassiliou E. Gastric neuroendocrine tumors: biology and management. Ann Gastroenterol 2005; 18: 127-40.

9. Falchetti A. Genetic screening for multiple endocrine neoplasia syndrome type 1 (MEN-1): when and how. F1000 Med Rep 2010; 2: 14.

10. Tsikitis VL, Wertheim BC, Guerrero MA. Trends of incidence and survival of gastrointestinal neuroendocrine tumors in the United States: a seer analysis. J Cancer 2012; 3: 292-302.

11. Li TT, Qiu F, Qian ZR, Wan J, Qi XK, Wu BY. Classification, clinicopathologic features and treatment of gastric neuroendocrine tumors. World J Gastroenterol 2014; 20: 118-25.

12. Sato Y, Hashimoto S, Mizuno K, Takeuchi M, Terai S. Management of gastric and duodenal neuroendocrine tumors. World J Gastroenterol 2016; 22: 6817-28. 